\title{
A soft aquatic actuator for unsteady peak power amplification
}

\author{
Francesco Giorgio-Serchi ${ }^{1}$, Artur K. Lidtke ${ }^{2}$ and Gabriel D. Weymouth ${ }^{2}$,
}

\begin{abstract}
A soft hydraulic actuator is presented which uses elastic energy storage for the purpose of pulsed-jet propulsion of soft unmanned underwater vehicles. The actuator consists of a flexible membrane which can be inflated using a micro-pump and whose elastic potential energy may be released on demand using a controllable valve, in a manner inspired by the swimming of squids and octopuses. It is shown that for equivalent initial elastic energy, the drop in peak thrust is linearly proportional to the decrease in nozzle cross section. Peak hydraulic power amplification of the soft actuator of approximately $75 \%$ is achieved with respect to that of the driving pump, confirming that passive elasticity can be exploited in aquatic propulsion to replicate the explosive motion skills of agile sea-dwelling creatures.
\end{abstract}

Index Terms-Soft actuators, hydraulic actuators, soft robots, bioinspired robots, aquatic robots, soft locomotion, variable stiffness actuators, explosive motion.

\section{INTRODUCTION}

D ESPITE the widespread employment of Unmanned Underwater Vehicles (UUVs), the degree of automation in the offshore sector remains sparse largely due to technological limitations. The increased complexity of operations which UUVs are tasked with has pushed for an incremental improvement of the capabilities and efficiency of existing vehicles [1]. Nonetheless, the low predictability and unsafe nature of the aquatic environment, and the complexity of navigation in unstructured submerged scenarios represent overwhelming constrains in the development of innovative UUVs.

In an attempt to address these limitations, disruptive design criteria based on the inspiration from sea dwelling organisms have been adopted in order to enhance propulsive performance, manoeuvrability and sensing capability [2], [3]. In addition, the pursue of new robotics systems with high degree of compliance stems from the realization that safe physical interaction with the environment and navigation in highly perturbed, complex environments can be managed by the inherent structural flexibility of the system rather than by increasing the accuracy of its control [4]. Among the many biological sources of inspiration [5], cephalopods (i.e. squids and octopuses) are peculiar in that they combine extreme bodily softness with unsurpassed swimming agility, thus embodying a unique synthesis of two highly sought for features in novel aquatic vehicles design, [6]. From a structural perspective these organisms represent the quintessential source of inspiration for a soft-bodied robot since they are almost entirely devoid of a rigid skeleton;

${ }^{1} \mathrm{~F}$. Giorgio-Serchi is with the School of Engineering, University of Edinburgh, Edinburgh, UK, f.giorgio-serchided.ac.uk; ${ }^{2} \mathrm{~A} . \mathrm{K}$. Lidtke and G. D. Weymouth are with the Faculty of Engineering and the Environment, University of Southampton, Southampton, UK however, it is their propulsion mechanics which make them remarkable. Cephalopods propel themselves by the periodic formation of pulsed jets of water which they generate by rhythmically expanding and collapsing an elastic cavity of their body [7]. They are able to finely regulate their size change in order to tune the speed and volume of the issuing fluid. This has significant implications for the impulse generated per pulsation as well as for the overall swimming efficiency. At low stroke ratios the expelled jet forms a vortex ring in combination with which a thrust peak is observed [8], [9]. At high stroke ratios the volumetric change of the organisms is such that prominent added-mass variation effects may occur [10], [11], thus giving rise to a large positive contribution to the total jetting impulse [12]. These features make the shape-changing pulsed-jet propulsion of cephalopods an ideal case study in augmented manoeuvrability and efficiency for the purpose of Soft Unmanned Underwater Vehicle (SUUV) design.

The present work describes the development of a new soft hydraulic actuator capable of replicating both the structural features as well as the explosive motion skills of cephalopods. Unsteady characterisation of the thrust force and turning moment developed by the vectoring jet of the robot is carried out

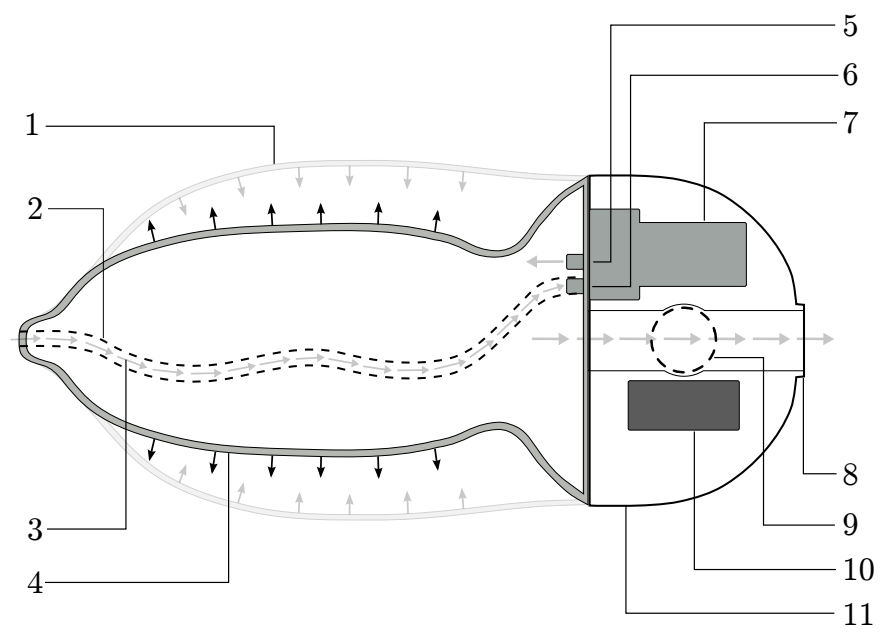

Fig. 1: Schematic depiction of the fixed compliance soft hydraulic actuator and its working principle. (1) - elastic membrane in the inflated state, (2) - internal hose for fluid ingestion, (3) - fluid flow during suction, (4) - elastic membrane in the collapsed state, (5) - pump exhaust, (6) - pump inlet, (7) centrifugal pump, (8) - nozzle, (9) - ball valve, (10) - servo motor for valve control, (11) - rigid compartment. 


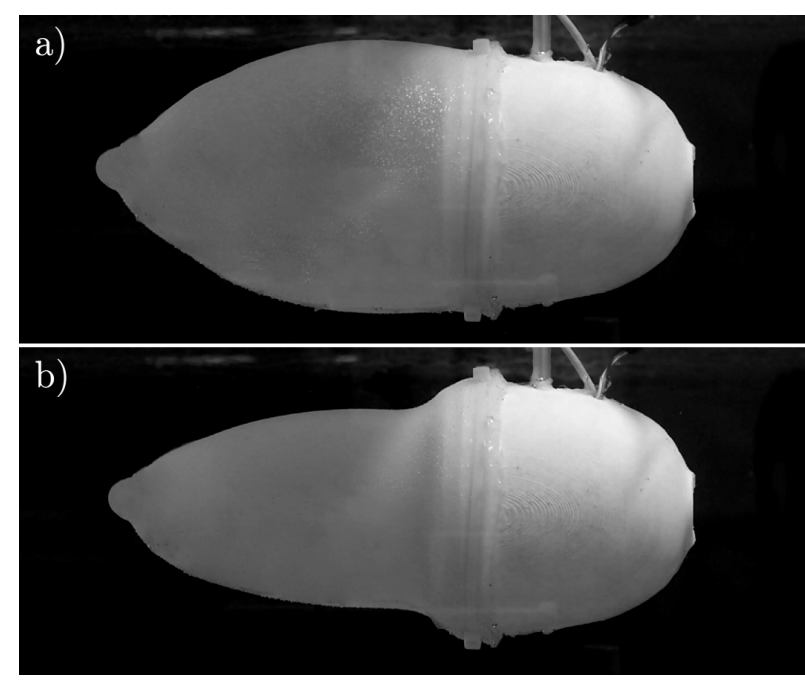

Fig. 2: Snapshots of the actuator during one single pulsation: (a) inflated configuration, $1.00 \mathrm{~L}$ and (b) deflated configuration, $0.62 \mathrm{~L}$.

using a two degree of freedom force measurement apparatus. Results of the measurements carried out for several different exit nozzle configurations are employed to provide a clear image of performance characteristics of the actuator and comment on its applicability to underwater vehicle propulsion.

\section{VEHICLE DESIGN}

Utilisation of passive elasticity for power enhancement can be enabled by means of flexible components which harness potential energy generated at a low rate and later release this energy in an abrupt fashion [13]. Here we present a soft fluidic actuator which relies on elastic potential energy storage for driving the pulsated routine of a cephalopod-inspired aquatic vehicle.

The actuator, schematically depicted in Fig. 1 and shown in Fig. 2, consists of an elastic hollow membrane fitted onto a rigid compartment which contains a valve and a pump. In analogy with the pulsed-jetting routine of cephalopods, the actuator working principle entails a periodic sequence of expansion and collapse of the elastic component. During inflation, ambient fluid is pushed into the elastic cavity of the vehicle by the pump across an inlet located at the bow of the vehicle. This charges the membrane with elastic potential energy while outflow of the internal fluid is prevented at the nozzle exit by the valve, Fig. 2(a). Once inflated, the valve is opened, enabling the sudden collapse of the membrane, Fig. 2(b), which ultimately results in the internal fluid issuing across the nozzle located at the stern and giving rise to a net forward thrust.

In the current prototype the membrane is a $250 \mathrm{~mm}$ axisymmetric ellipsoid truncated at $75 \%$ of its length and $4 \mathrm{~mm}$ thick. At the truncation location, the membrane is fitted onto a $3 \mathrm{D}$ printed housing which hosts the outflow valve and the pump. A centrifugal M510 series TCS-micropump,with a maximum flow-rate of $8.7 \mathrm{~L} / \mathrm{min}$ and a maximum rated head pressure of $586 \mathrm{mbar}$, is employed to inflate the elastic
TABLE I: Key design parameters of the different nozzles tested. Diameter is defined at the outlet plane and direction is measured with respect to the vehicle axis.

\begin{tabular}{ccc}
\hline Nozzle & Inside diameter [mm] & Outlet direction [deg] \\
\hline Baseline (no nozzle) & 20 & 0 \\
Medium & 15 & 0 \\
Small & 10 & 0 \\
& & \\
15 deg & 15 & 15 \\
$30 \mathrm{deg}$ & 15 & 30 \\
$45 \mathrm{deg}$ & 15 & 45 \\
\hline
\end{tabular}

membrane. The outflow across the nozzle is regulated by a 3D printed ball-valve actuated by a Traxxas 2080 waterproof servo, via coupled spur gears which allow for a quicker operation of the valve. The pump is powered using a $12 \mathrm{~V}$ DC power supply and its rpm is adjusted using an Electronic Speed Controller (ESC). The ESC and the valve servo are managed by an Arduino micro-controller placed outside the robot.

\section{Methodology}

The actuator is characterised by assessing the relationship between the elastic deformation of the system and the impulse output and by estimating vehicle manoeuvrability via the measurement of the turning moment due to jet vector-thrusting. To do so, the tests are executed with the vehicle held still and measuring the resultant forces generated by several nozzle configurations while the actuator is commanded to perform repeated sequences of pulsed-jetting at various inflation dutycycles.

Two main sets of nozzles are considered: two straight nozzles with internal diameters of $10 \mathrm{~mm}$ and $15 \mathrm{~mm}$, and three deflected nozzles at $15^{\circ}, 30^{\circ}$ and $45^{\circ}$ angles. These are summarised in Table I and depicted in Fig. 3. Each test consists in the pump continuously operating for $1.0,2.5,5.0,7.5,10.0$, $12.5,15.0,17.5$ or $20.0 \mathrm{sec}$ and then resting idle for the following 5.0 seconds, during which the valve is open, letting the flow issue across the nozzle. This routine is repeated for ten cycles, eventually providing a force measurement averaged over the whole sequence. An example of the raw data from this routine executed with a $15.0 \mathrm{sec}$ inflation time is depicted in Fig. 4 (black line).

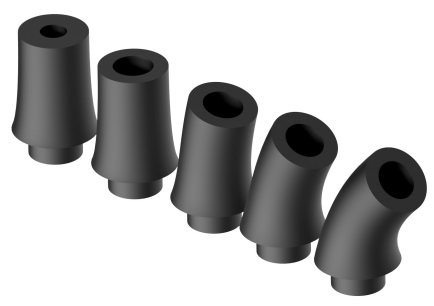

Fig. 3: Nozzles used to modify the propulsive characteristics of the baseline vehicle. Two left-most nozzles are straight and of varying internal diameter, the remaining ones are angled to deflect the direction of the jet and induce a turning moment. 


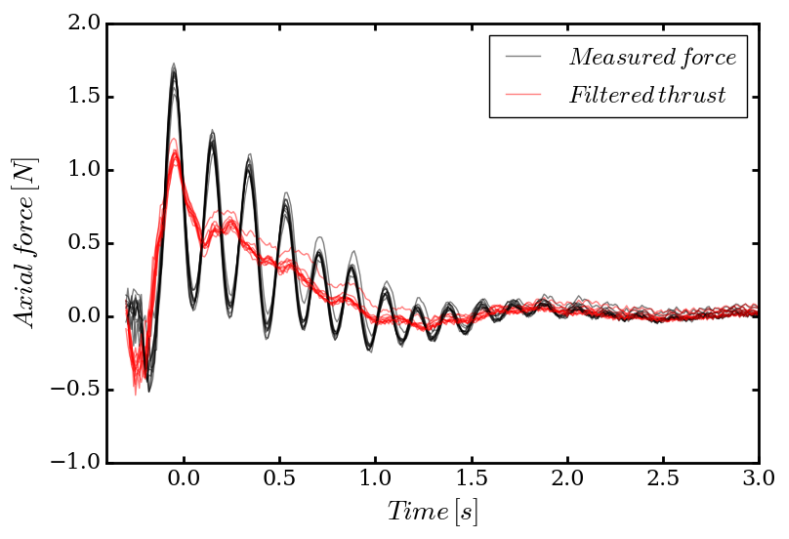

Fig. 4: Example of the time history of the measured force (black) and inferred thrust (red) following a $15.0 \mathrm{sec}$ dutycycle. The signals show data measured during a single $5.0 \mathrm{sec}$ jet discharge over ten repetitions of the same test. Measurements from all tests are aligned in time based on the occurrence of the first peak. Data in the negative part of the time axis corresponds to the period between the opening of the valve and the occurrence of the maximum response measured. Filtered data (red) have been obtained by applying a bandstop filter at the estimated natural frequency of the system.

\section{A. Force measurement system}

The measurement apparatus is described in detail in [14] and consists of a platform suspended over an experimental water tank and held in place by aluminium bars. This is appended with the force measuring unit, composed of two strain gaugeinstrumented load cells mounted in a perpendicular orientation to the horizontal plane of the robot and arranged at a known relative distance from the axis of the robot. As a forward force is exerted on the system by the issuing fluid, the total shear of the load cells yields the linear thrust produced by the vehicle, while the differential of the two shear readings is calibrated to output the yaw moment. Thus, following appropriate calibration [14], each couple of transducers enables the acquisition of accurate unsteady loads in 2-DOF. The transducers are attached to two aluminium blocks, one of which is connected to the supporting plate, with the other housing an $8 \mathrm{~mm}$ vertical aluminium rod that links the force measuring system with the submerged robot. Each of the load cells is excited with $5 \mathrm{~V}$ DC voltage, which is transformed into a voltage drop linearly proportional to the applied load via the use of a Wheatstone bridge of strain gauges. The use of an INA125P integrated amplifier circuits allows a $100 \mathrm{~Hz}$ acquisition rate.

\section{B. Recovery of the thrust signal}

Measurement of unsteady, pulsating thrust bears significant difficulties stemming from the fact that the system comprising of the measurement apparatus and the soft aquatic actuator vibrates at its own natural frequency when subject to the impulsive load generated by the jet. This, in turn, leads to the measured force signal consisting of both the true thrust time history and an oscillatory component associated with the dynamics of the whole rig (i.e the measurement apparatus

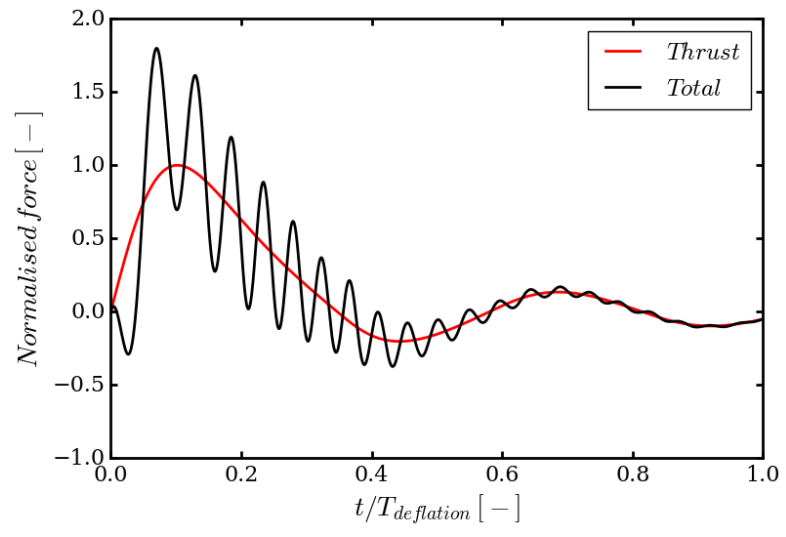

Fig. 5: Normalised thrust and transducer signal simulated using a numerical model representing the current system. Thrust is the actual forward force developed by the actuator and the Total signal represents the ensemble of thrust and force arising due to oscillation of the system, which is what would be measured in the experiments.

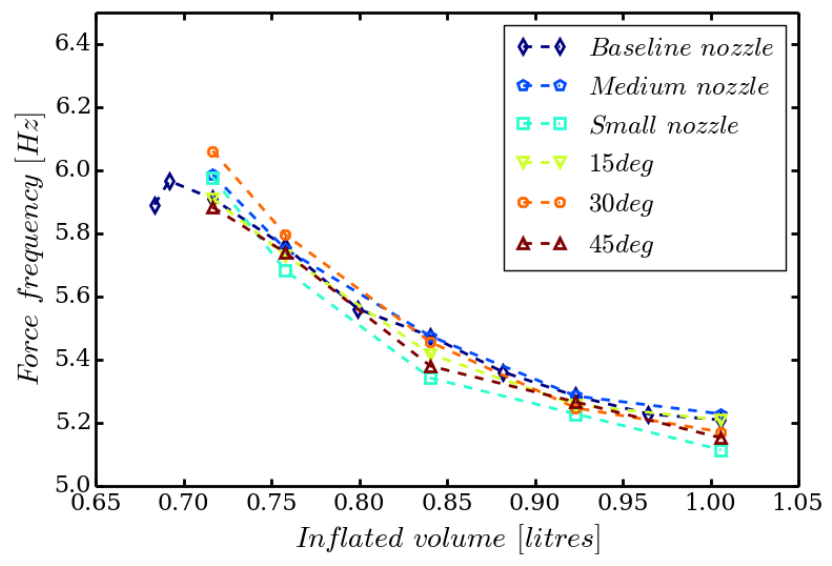

Fig. 6: Dominant frequency of the thrust signal as a function of different robot configurations and prescribed inflation time.

and the actuator). In Fig. 4 the recorded signal (black line) from ten successive pulsations overlapped one onto the other is portrayed, showing that the recorded signal appears strongly affected by a periodic force component. In order to ascertain that this signal can be used to derive the actual thrust, a simplified dynamic model of the whole apparatus (actuator and measurement system) was employed. The model, described in [15], treats the apparatus (i.e. the ensemble of the measuring rig and the pulsed-jet mass-varying actutor) as a system of two coupled oscillators. One captures the impulsive force generated by the pulsed-jet by accounting for the dynamics of a water-filled elastic membrane which pulsates along its minor semi-axis under the effect of an initial deformation from its unstrained state. The other describes the vibration of the system in the direction tangential to the jet by treating the measurement rig as a cantilever subject to deflection due to an impulsive force (i.e. the expulsion of fluid from the membrane) exerted at its free end. By prescribing an initial inflation of the membrane, the thrust, red line in Fig. 5, and the force measured 
at the fixed end of the cantilever, black line in Fig. 5, can be simulated in the time-domain. The model offers a qualitative description of the thrust signal which is to be expected from filtering the raw measured data.

Performing FFT analysis of all tests at various inflation rates allows derivation of the natural frequency of the system, presented in Fig. 6. The data show that natural frequencies obtained in this way are dependent exclusively on the maximum inflated volume. By treating the measured signals with a bandstop filter in the bandwidth of the natural frequencies observed in Fig. 6, the profiles obtained appear as in Fig. 4 (red lines). Comparing them to the simulated results shows that use of bandstop filtering yields only partial under-prediction of the real thrust and hence it can be employed to perform further analysis of the actuator.

\section{RESUlts}

\section{A. Accumulated potential energy}

The working routine of this actuator relies on accumulating elastic potential energy in the membrane in order to later transform it into kinetic energy of the issuing fluid. Peak fluid volume obtained for each duty cycle may thus be used as a measure of stored potential energy. Inflated volume of the robot was measured using contour thresholding of video frames for different period of pump operation and was found to follow a linear trend of 0.0165 litres per second of actuation, equal to approximately $11 \%$ of the rated flow rate of the pump.

Not withstanding viscous losses, the elastic potential energy stored in the membrane yields an estimate of the jet speed and offers the rationale for a simple scaling of the jet impulse. The elastic potential energy is expressed as,

$$
P E=\frac{1}{2} k_{0} b^{2}
$$

where $b$ is the extension of the minor semi-axis of the membrane and $k_{0}$ is the elastic constant of the membrane. When the valve is opened, this potential energy is transformed into kinetic energy of the fluid,

$$
P E \approx K E=\frac{1}{2} m_{f} v_{f}^{2}
$$

where $m_{f}$ represents the fluid inertial terms within the deforming membrane and $v_{f}$ the fluid velocity at the nozzle exit plane. Hence the speed at which the fluid is accelerated is

$$
v_{f} \approx b \sqrt{\frac{k_{0}}{m_{f}}}
$$

This suggests that a quasi-linear relationship between the amount of stretching of the membrane and the jet flow is to be expected. By noticing that the jet thrust $\tau \propto v_{f}^{2}$, eq. 3 also shows that a leading term in the thrust produced is represented by the inertial effect of the internal fluid, as clearly illustrated by the comparison of test performed with nozzle of different diameters in Fig. 7.

By defining a reference volume $V=\frac{4}{3} \pi L b^{2}$ and timescale of the pulsation $T$, and given the major semi-axis of the membrane $L$, then

$$
v_{f} \propto \dot{V} \rightarrow \overline{v_{f}} \propto \frac{\Delta V}{T}
$$

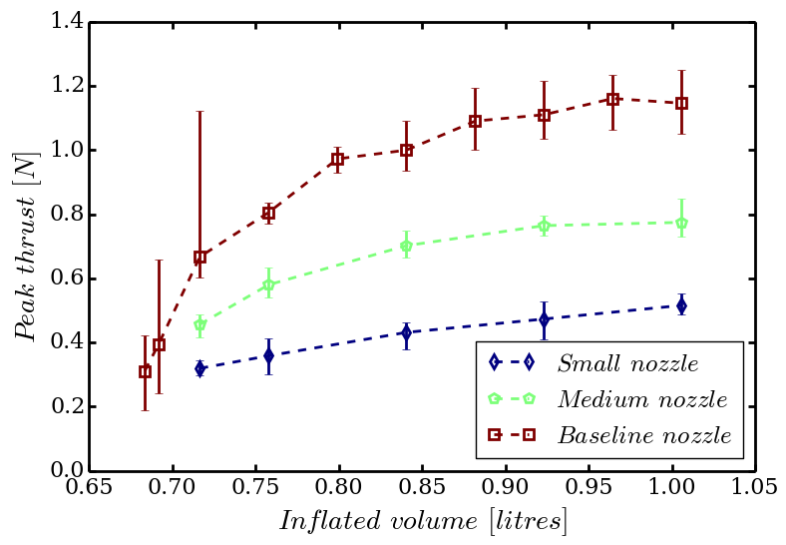

(a) Peak thrust

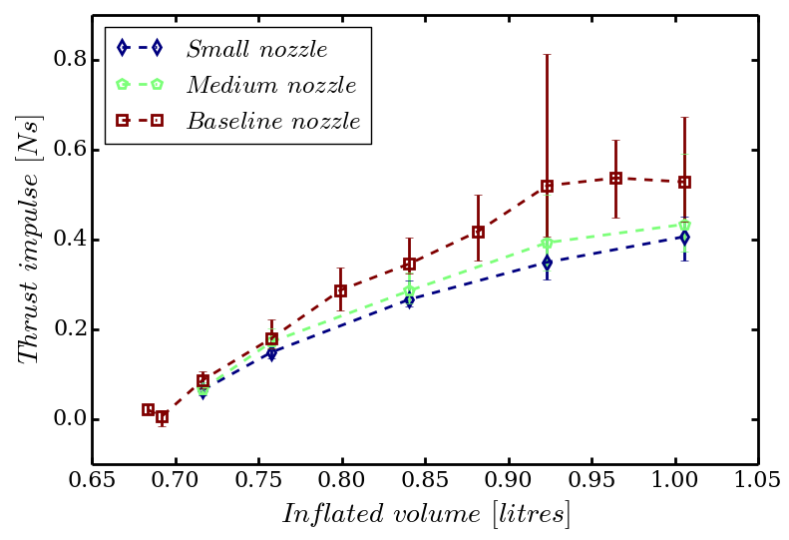

(b) Developed impulse

Fig. 7: Effect of outlet diameter of the straight nozzles on the thrust. Markers on each dashed line follow the mean of the recorded duty cycles and error bars indicate the maximum deviation from the mean observed in the collected data.

where $\overline{v_{f}}$ is the time-averaged flow rate across the nozzle over the pulsation timescale. This confirms that

$$
K E=\int_{0}^{T} \tau v_{f} d t=\rho A \int_{0}^{T} v_{f}^{3} d t \propto \frac{\Delta V^{3}}{T^{2}}
$$

with $\rho$ and $A$ the fluid density and nozzle cross section. Hence, given that $P E \propto \Delta V$ and since $P E \approx K E$, then $T \propto \Delta V$ (i.e. the time of flow discharge is proportional to the volume variation) which yields the impulse to be,

$$
I=\int_{0}^{T} \tau d t=\rho A \int_{0}^{T} v_{f}^{2} d t \propto \frac{\Delta V^{2}}{T} \approx \Delta V
$$

which qualitatively describes the trend reported in Fig. 7(b).

\section{B. Effect of nozzle diameter}

The thrust generated by mass-varying system is expected to grow quadratically with the reduction of nozzle radius. However, the inertial effect $m_{f}$ are largely dependent on the confinement effect of the fluid at the nozzle entrance plane. This gives rise to a non-linear relationship between thrust and nozzle diameter for systems driven by passive elasticity such as the one considered here. The effects of 
nozzle diameter reduction on peak thrust are presented in Fig. 7(a). In accordance with the above considerations and with reference to Eq. 3, a trend is observed where smaller nozzles produce smaller peak thrust. However, the recorded impulse, Fig. 7(b), appears to be less affected by the diameter of the outlet, with all of the nozzles predicting almost identical results for the lower one third of inflation volumes. This is because peak thrust has been shown to depend on $m_{f}$, where larger nozzles have lower internal added-mass effects and hence manifest fast discharge rate. However, the impulse depends more conspicuously on the viscous losses inside the membrane which, for the case tested, are comparable across all nozzle configurations.

\section{Effect of nozzle angle}

The chance to use vector thrusting as the default mode of manoeuvring is critical to the employment of these kind of vehicles in real world scenarios. In order to fully exploit pulsedjetting for agile manoeuvring, the vehicle must not be reliant on control surfaces. Hence, the use of bent nozzles as a mean to vector the jet to achieve enhanced manoeuvrability is tested here for the first time. Fig. 8 presents the effect of nozzle angle on the developed peak thrust and yaw moment amplitudes. As expected, the higher the angle of the nozzle, the larger the induced moment and the lower the forward thrust. The test seem to suggest that an optimal nozzle configuration exists, in this case the $30^{\circ}$ deflection, where high yaw moment and high thrust coexist. The experiments also show a unexpected trend where higher flow rate do not yield a proportional torque.

\section{Discussion}

The results presented in section IV provide a first insight into the mechanism of thrust generation from an elastically charged membrane. The dependence of the peak thrust on the amount of potential energy stored in the elastic walls suggests that the employment of a hydraulic pump as the underlying inflation mechanism brings significant limitations. The effectiveness of this mode of actuation lies in either the chance to quickly inflate the membrane to a lesser extent or slowly inflate it to its maximum capacity. The former case is constrained by the fact that, below a certain threshold, useful thrust output drops dramatically, as evident from Fig. 7. The latter case, however, may not be suitable in attaining a steady swimming pace, given the extensive time lag between one pulsation and the next. It is important to underline that these tests are performed with the actuator held still, thus neglecting all contributions from added-mass-variation term [12], which can significantly enhance thrust and impulse.

The comparison of the hydraulic power generated by the soft actuator with respect to the hydraulic power generated by the pump confirms that the exploitation of elastic energy storage plays a substantial role in amplifying the peak power, as seen in Fig. 9.

This was computed as $P=\tau \cdot U_{j e t}$, with the jet velocity, $U_{\text {jet }}$, being estimated based on known nozzle parameters. Power amplification in aquatic systems which exploit passive compliance is significantly affected by the fluid inertial effects

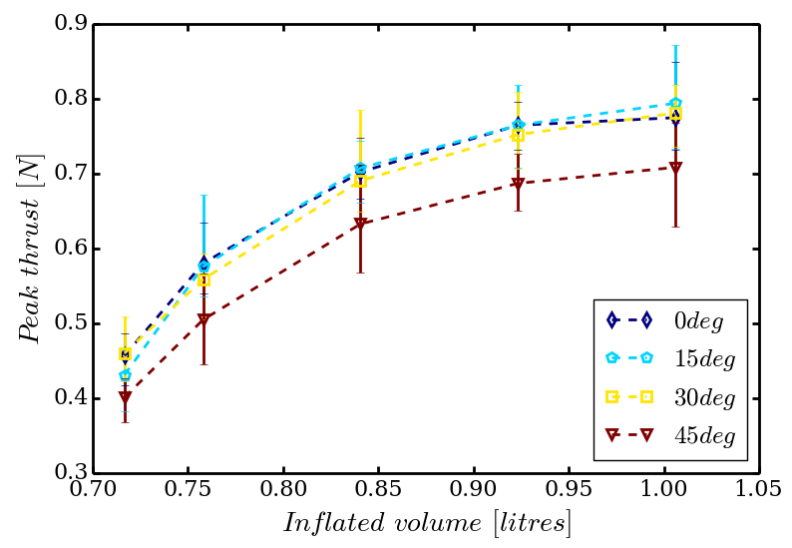

(a) Thrust

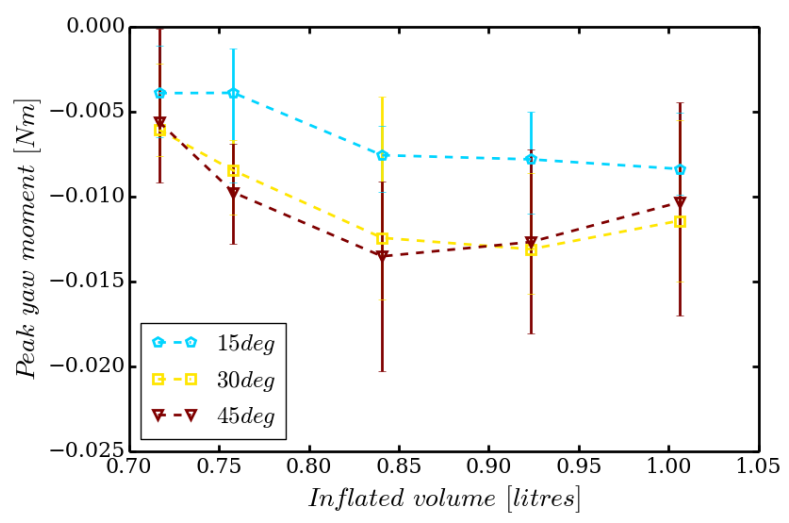

(b) Yaw moment

Fig. 8: Effect of the nozzle angle on the peak thrust and yaw moment developed by the vehicle.

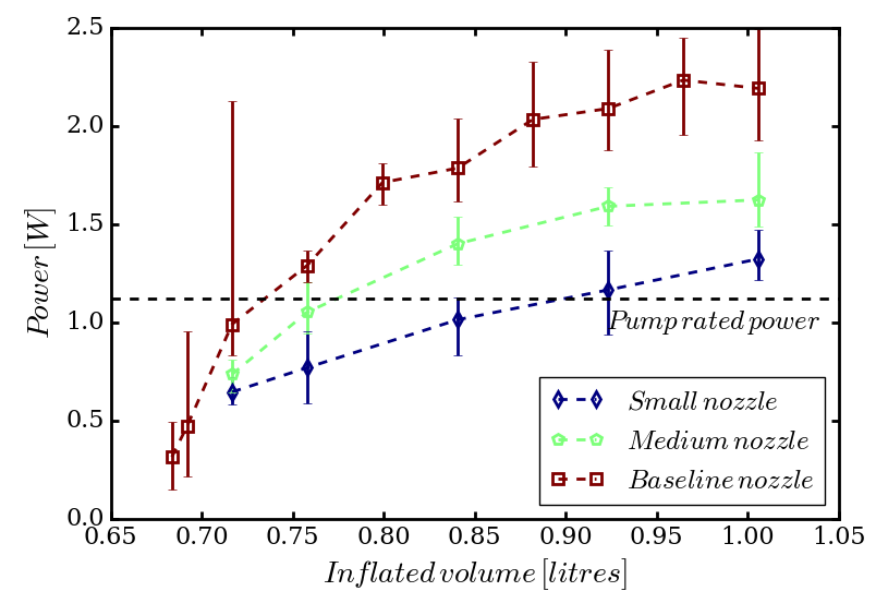

Fig. 9: Hydraulic peak powers of the soft actuator tested with the three nozzle configurations and that of the centrifugal pump (power rated at $1.5 \mathrm{~L} / \mathrm{min}$ ).

which ultimately control the rate of discharge of the elastic potential energy, Fig. 9. Nonetheless, hydraulic peak power output from the soft actuator can be as large as $75 \%$ of the centrifugal pump when operating at $1.5 \mathrm{~L} / \mathrm{min}$. 
Finally, the employment of a steerable nozzle as a means of controlling trajectory of the vehicle in three dimensions has been shown to represent a viable option. While it is not feasible to accurately predict a length scale for the turning radius based on the measured unsteady torque alone, the results of Fig. 8 bring evidence of two key factors. In a mass-varying system such as the one considered at present, the displacement of the centre of mass during deflation plays a remarkable role in negatively affecting the stability during turning manoeuvres. This is apparent in the non-linearity of the yaw moment recorded at the higher inflation rates and in the amount of underlying noise observed in these measurements. In addition, the response of linear and angular component to jet orientation shows that nozzle design can be optimized to yield optimal performance within a narrow range of deflection angles.

\section{CONCLUSIONS}

A novel soft hydraulic actuator for aquatic pulsed-jet propulsion of SUUVs is presented. The actuator is, to a large extent, flexible, making it a valid candidate in the search for new design principles apt for operations in complex environments. Lack of external moving parts also participates in making it suitable for navigation in unstructured and cramped submerged scenarios or among fields of debris. The self contained design and the employment of a small-scale centrifugal pump enables the actuator to generate low noise levels and promises an ultimate independent vehicle design to be difficult to detect by conventional means. The actuator presented can be employed as a stand alone vehicle similar to those in [16], [17]. In addition, its implementation in various configurations within larger vehicles has been explored in details in [18].

The present set of tests provides a first clear characterization of the mechanism of thrust generation via the exploitation of passive elasticity. The analysis demonstrates that peak power amplification is indeed achievable and can be exploited to perform a broad range of swimming modes ranging from lowfrequency, high-thrust pulsations to high-frequency, low-thrust pulsations.

Ultimately, the current set of tests represents a benchmark for model validation which will be used to fine-tuning the key design parameters in order to achieve a stable, self-propelled prototype.

\section{ACKNOWLEDGMENT}

This work was supported by the Lloyd's Register Foundation, the Natural Environment Research Council (grant NE/P003966/1), the SMMI-HEIF grant of the Southampton Marine and Maritime Institute and the EPSRC Robotics and Artificial Intelligence ORCA Hub (grant No. EP/R026173/1).

\section{REFERENCES}

[1] Y. Shi, C. Shen, H. Fang, and H. Li, "Advanced control in marine mechatronic systems: A survey," IEEE/ASME Transactions on Mechatronics, vol. 22, no. 3, pp. 1121-1131, June 2017.

[2] J. Yu, C. Zhang, and L. Liu, "Design and control of a single-motoractuated robotic fish capable of fast swimming and maneuverability," IEEE/ASME Transactions on Mechatronics, vol. 21, no. 3, pp. 17111719 , June 2016.
[3] J. Yu, Z. Su, Z. Wu, and M. Tan, "Development of a fast-swimming dolphin robot capable of leaping," IEEE/ASME Transactions on Mechatronics, vol. 21, no. 5, pp. 2307-2316, Oct 2016.

[4] R. Woodman, A. F. Winfield, C. Harper, and M. Fraser, "Building safer robots: Safety driven control," The International Journal of Robotics Research, vol. 31, pp. 1603-1626, 2012.

[5] M. Calisti, G. Picardi, and C. Laschi, "Fundamentals of soft robot locomotion," Journal of The Royal Society Interface, vol. 14, no. 130, 2017. [Online]. Available: http://rsif.royalsocietypublishing.org/content/ $14 / 130 / 20170101$

[6] F. Giorgio-Serchi, A. Arienti, F. Corucci, M. Giorelli, and C. Laschi, "Hybrid parameter identification of a multi-modal undwater soft robot," Bioinspiration \& Biomimetics, vol. 12, no. 2, p. 025007, 2017.

[7] W. Johnson, P. D. Soden, and E. R. Trueman, "A study in met propulsion: an analysis of the motion of the squid, Loligo Vulgaris," Journal of Experimental Biology, vol. 56, pp. 155-165, 1972.

[8] P. S. Krueger and M. Gharib, "The significance of vortex ring formation to the impulse and thrust of starting jet," Physics of Fluid, vol. 15, pp. 1271-1281, 2003

[9] M. Krieg and K. Mohseni, "Modelling circulation, impulse and kinetic energy of starting jets with non-zero radial velocity," Journal of Fluid Mechanics, vol. 79, pp. 488-526, 2013.

[10] G. Weymouth and M. Triantafyllou, "Ultra-fast escape of a deformable jet-propelled body," Journal of Fluid Mechanics, vol. 721, pp. 367-385, 2013.

[11] F. Giorgio-Serchi and G. D. Weymouth, "Drag cancellation by addedmass pumping," Journal of Fluid Mechanics, vol. 798, 2016.

[12] G. Weymouth, V. Subramaniam, and M. Triantafyllou, "Ultra-fast escape maneuver of an octopus-inspired robot," Bioinspiration \& Biomimetics, vol. 10, pp. 1-7, 2015.

[13] S. Wolf, G. Grioli, O. Eiberger, W. Friedl, M. Grebenstein, H. Hppner, E. Burdet, D. G. Caldwell, R. Carloni, M. G. Catalano, D. Lefeber, S. Stramigioli, N. Tsagarakis, M. V. Damme, R. V. Ham, B. Vanderborght, L. C. Visser, A. Bicchi, and A. Albu-Schffer, "Variable stiffness actuators: Review on design and components," IEEE/ASME Transactions on Mechatronics, vol. 21, no. 5, pp. 2418-2430, Oct 2016.

[14] A. Lidtke, F. Giorgio-Serchi, and G. D. Weymouth, "A low-cost experimental rig for multi-dof unsteady thrust measurements of aquatic bioinspired soft robots," in IEEE RAS RoboSoft, Livorno, Italy, April 2018.

[15] G. Weymouth and F. Giorgio-Serchi, "Analytic modeling of a sizechanging swimmer," in IUTAM Symposium on Critical flow dynamics involving moving/deformable structures with design applications, Santorini, Greece, June 2018.

[16] F. Renda, F. Giorgio-Serchi, F. Boyer, and C. Laschi, "Structural dynamics of a pulsed-jet propulsion system for underwater soft robots," International Journal of Advanced Robotic Systems, vol. 12, no. 6, p. 68, 2015. [Online]. Available: https://doi.org/10.5772/60143

[17] F. Giorgio-Serchi, A. Arienti, and C. Laschi, "Underwater soft-bodied pulsed-jet thrusters: Actuator modeling and performance profiling," International Journal of Robotics Research, 2016.

[18] F. Renda, F. Giorgio-Serchi, F. Boyer, C. Laschi, J. Dias, and L. Seneviratne, "A unified multi-soft-body dynamic model for underwater soft robots," The International Journal of Robotics Research, vol. 0, no. 0, p. 0278364918769992, 0. [Online]. Available: https://doi.org/10.1177/0278364918769992

Francesco Giorgio-Serchi is a Chancellor Fellow (lecturer) at the University of Edinburgh. He holds an MSc from the University of Pisa in Marine Technologies and a PhD in Computational Fluid Dynamics from the University of Leeds. His work entails the coupled fluid-solid interaction of deformable bodies with the purpose of designing bioinspired aquatic vehicles with enhanced manoeuvrability and efficiency.

Artur K. Lidtke graduated as a naval architect and worked in the field of marine propeller design. He subsequently obtained a $\mathrm{PhD}$ from the University of Southampton on numerical modelling of propeller noise but has since been working on hydrodynamic design of underwater gliders.

Gabriel D. Weymouth is an Associate Professor at the University of Southampton. He is also an affiliate of the Massachusetts Institute of Technology in the Center for Ocean Engineering. His research has two focuses: the development of fast, accurate, and robust computational methods for fluid/structure interactions; and the application of those techniques to interesting new problems in naval architecture and underwater robotics. 